\title{
PRIME POWER GROUPS IN WHICH EVERY COMMUTATOR OF PRIME ORDER IS INVARIANT*
}

\author{
BY

\section{WILLIAM BENJAMIN FITE}

In this paper I represent by $G$ a group of order $p^{m}$ ( $p$ an odd prime) and class $k$; by $H_{j}$ the $j$ th central of $\dagger G$; and by $G^{\prime}$ the first cogredient of $G$. The term " $i$ th commutator" is used in the sense explained in an earlier paper. $\ddagger$

If the central of $G^{\prime}$ is cyclic, $H_{3}$ must contain an operation $s$ that is not contained in $H_{2}$ whose $p$ th power is contained in $H_{1}$, since otherwise the quotient group $H_{3} / H_{1}$, which is the second central of $G^{\prime}$, would contain only one subgroup of order $p$, and would therefore be cyclic. $\S$ But this is impossible.\| Also $G$ must contain an operation, as $A$, such that $A^{-1} s A=s t$, where $t$ is contained in $H_{2}$, but not in $H_{1}$. Moreover if $s^{-1} t s=t h, h$ is invariant in $G$ and $s^{-p} t s^{p}=t h^{p}=t$, since $s^{p}$ is invariant in $G$. Hence the order of $h$ does not exceed $p$. It follows that $\uparrow(s t)^{p}=s^{p} t^{p} h^{p(p-1) / 2}=s^{p} t^{p}$. Hence $A^{-1} s^{p} A=(s t)^{p}=s^{p} t^{p}$, and $t^{p}=1$. This proves that if $G^{\prime}$ has a cyclic central, $\mathrm{H}_{2}$ must contain a commutator of order $p$ that is not invariant in $G$.

If now any cogredient of $G$, as the $i$ th $(1<i<k-1)$, contains a cyclic central, $H_{i+1} / H_{i}$ must be cyclic; and it follows from what has just been proved that there must be contained in $H_{i+1}$ a commutator $t_{i+1}$ which is not contained in $H_{i}$, but whose $p$ th power is contained in $H_{i-1}$. There must then be some operation of $G$, as $A_{i+1}$, such that

$$
A_{i+1}^{-1} t_{i+1} A_{i+1}=t_{i+1} t_{i}
$$

where $t_{i}$ is a second commutator contained in $H_{i}$, but not in $H_{i-1}$, and $t_{i}^{p}$ is contained in $H_{i-2}$. If $i>2$, there must be an operation $A_{i}$ such that

$$
A_{i}^{-1} t_{i} A_{i}=t_{i} t_{i-1},
$$

* Presented to the Society at the Providence meeting, September 9, 1914.

$\dagger$ This notation is that of Burnside's Theory of Groups of Finite Order, first edition, p. 62, and not that of the second edition, p. 120. Cf. de Séguier, Éléments de la théorie des groupes abstraits, p. 87.

$\ddagger$ Fite, these Trans a c tion s, vol. 7 (1906), p. 61 .

$\S$ Burnside, loc. cit., second edition, p. 131.

\|Fite, these Transaction s, vol. 15 (1914), p. 48.

If Fite, these Transactions, vol. 3 (1902), p. 337. 
where $t_{i-1}$ is a third commutator contained in $H_{i-1}$, but not in $H_{i-2}$, and $t_{i-1}^{p}$ is contained in $H_{i-3}$. We can continue this argument until we arrive at an $i$ th commutator of order $p$ that is contained in $H_{2}$ but not in $H_{1}$. Hence if any cogredient of $G$, as the ith, has a cyclic central, $\mathrm{H}_{2}$ must contain an ith commutator of order $p$ that is not invariant in $G$.

If every $i$ th $(i<k-1)$ commutator of order $p$ is invariant in $G$, as is the case, for example, when the $i$ th commutator subgroup is cyclic, neither the $i$ th cogredient nor any succeeding cogredient can have a cyclic central. Moreover there is no operation of $H_{i+2}$ that is not contained in $H_{i+1}$ whose $p$ th power is contained in $H_{i}$. For if $s$ were such an operation, there would be an operation, $A$, of $G$ such that

$$
A^{-1} s A=s t_{i+1},
$$

where $t_{i+1}$ is a commutator contained in $H_{i+1}$, but not in $H_{i}$. It follows from this that

$$
A^{-1} s^{p} A=s^{p} t_{i+1}^{p} t
$$

where $t$ is contained in $H_{i-1}$, as may be seen by applying an argument similar to the one on page 134 to the corresponding operations of the quotient group $G / H_{i-1}$. Hence, since $s^{p}$ is by hypothesis contained in $H_{i}, t_{i+1}^{p}$ must be contained in $H_{i-1}$. But the preceding argument shows that this is possible only in case $G$ contains a non-invariant $i$ th commutator of order $p$.

Since every $(i+1)$ th commutator is also an $i$ th commutator, ${ }^{*}$ there can be no operation of $H_{i+j}(j>2)$ that is not contained in $H_{i+j-1}$ whose $p$ th power is contained in $H_{i+j-2}$. Hence the order of every operation of $G$ that is not contained in $H_{k-1}$ must be at least $p^{k-i}$.

If we suppose that every $i$ th commutator that is contained in $H_{2}$ and whose order does not exceed $p^{r}(r>0)$ is invariant in $G$, we shall see that the facts just mentioned are particular phases of somewhat more general ones.

For if an operation $s$ of $H_{i+2}$ that is not contained in $H_{i+1}$ were such that its $p^{r}$ th power is contained in $H_{i}$, there would be an operation $A$ of $G$ such that

$$
A^{-1} s A=s t_{i+1},
$$

where $t_{i+1}$ is contained in $H_{i+1}$, but not in $H_{i}$. Moreover

$$
A^{-1} s^{p^{r}} A=s^{p^{r}} t_{i+1}^{p^{r}} t,
$$

where $t$ is contained in $H_{i-1}$. Since $s^{p^{r}}$ is contained in $H_{i}, t_{i+1}^{p r}$ must be contained in $H_{i-1}$. If $i=1$ this would be contrary to the hypothesis, since the order of the non-invariant first commutator $t_{i+1}$ would be equal to, or less than, $p^{r}$. If $i>1$, we can apply a similar argument to $t_{i+1}$ and continue the process until we arrive at a non-invariant $i$ th commutator that is contained

${ }^{*}$ Fite, these Transactions, vol. 7 (1906), p. 61. 
in $H_{2}$ and whose order does not exceed $p^{r}$. But by hypothesis there is no such commutator. Hence if every ith commutator of $G$ that is contained in $H_{2}$ and whose order does not exceed $p^{r}$ is invariant in $G$, the $p^{r}$ th power of an operation of $H_{i+2}$ that is not contained in $H_{i+1}$ cannot be contained in $H_{i}$.

It follows that the order of every operation of $H_{i+j}(j \geqq 2)$ that is not contained in $H_{i+j-1}$ must be greater than $p^{r(j-1)}$, and that every operation not contained in $H_{k-1}$ must be of order greater than $p^{r(k-i-1)}$. In particular, if every commutator whose order does not exceed $p^{r}$ is invariant, the order of every operation that is not contained in $H_{k-1}$ must be greater than $p^{r(k-2)}$.

The following statement follows from the preceding general discussion as an important special case: If every operation of $\mathrm{H}_{2}$ whose order does not exceed $p^{r}$ is invariant in $G$, every operation of $G$ whose order does not exceed $p^{r}$ is invariant.

If every $i$ th commutator of order $p$ that is contained in $H_{2}$ is invariant in $G$, no cogredient after the $i$ th one can have a central that is generated by more independent generators than the central of the immediately preceding cogredient. This can be seen by comparing first the number of independent generators of the central of a group $L$ of order a power of $p$ with the number of independent generators of the central of the first cogredient of $L$. Let $L_{j}$ and $L^{\prime}$ have the same significance with reference to $L$ that $H_{j}$ and $G^{\prime}$ respectively have with reference to $G$.

If the $p$ th power of some operation of $L_{2}$ that is not contained in $L_{1}$ is equal to the $p$ th power of some operation of $L_{1}$, there must be contained in $L_{2}$ an operation of order $p$ that is not contained in $L_{1}$. If there is no such operation in $L_{2}$, the $p$ th power of an operation of $L_{2}$ that corresponds to an operation of order $p$ of $L^{\prime}$ must be some one of the $p^{a-n_{1}}\left(p^{n_{1}}-1\right)$ operations of $L_{1}$ that are not $p$ th powers of operations of $L_{1}$. Here $p^{a}$ and $n_{1}$ represent respectively the order of $L_{1}$ and the number of its independent generators. If the central of $L^{\prime}$ is generated by $n$ independent generators, $L_{2}$ contains $p^{a}\left(p^{n}-1\right)$ operations that correspond to operations of order $p$ of the central of $L^{\prime}$. Moreover these operations can be divided into sets of $p^{n_{1}}$ operations each such that the operations of any set have the same $p$ th power, while two operations of different sets have different $p$ th powers. This follows from the fact that $L_{1}$ contains $p^{n_{1}}$ operations whose orders do not exceed $p$ and the assumption that every operation of $L_{2}$ not contained in $L_{1}$ is of order greater than $p$. Hence $L_{1}$ must contain $p^{a-n_{1}}\left(p^{n}-1\right)$ operations that are $p$ th powers of operations of $L_{2}$, but not $p$ th powers of operations of $L_{1}$. This would be impossible if $n>n_{1}$.

If now the central of the $(i+j)$ th cogredient of $G(j \geqq 1)$-that is, the central of $G / H_{i+j}$-were generated by $n$ independent generators, while the central of $G / H_{i+j-1}$ is generated by $n_{1}$ independent generators, where $n>n_{1}$, 
we should have the case just considered with $G / H_{i+j-1}$ for $L$. Hence the $p$ th power of some operation of $H_{i+j+1}$ not contained in $H_{i+j}$ would be contained in $H_{i+j-1}$. But this is impossible since every $i$ th commutator of order $p$ that is contained in $\mathrm{H}_{2}$ is invariant in $G$.

Suppose now that every commutator of $G$ that is contained in $H_{2}$ and whose order does not exceed $p^{r}$ is invariant in $G$, and that $G$ contains an invariant commutator of this order, but none of higher order. Then the $p^{r}$ th power of any operation of any central after the first one is contained in the preceding one. Moreover in any cogredient of $G$ the commutators in the second central whose orders do not exceed the order of every invariant commutator of this cogredient must themselves be invariant in this cogredient.

If $H_{k-1}$ is abelian let $s$ be any one of its operations and $A$ any operation of $G$ not contained in $H_{k-1}$. Then

$$
s^{-1} A s=A t,
$$

where $t$ is contained in, say, $H_{j}$, but not in $H_{j-1}$. Suppose that

$$
A^{-1} t A=t t_{1} \text {. }
$$

Then $t_{1}$ is contained in $H_{j-1}$. If now $A^{\prime}, t^{\prime}$, and $t_{1}^{\prime}$ are the operations of $G / H_{j-2}$ that correspond respectively to $A, t$, and $t_{1}$, then

$$
\left(A^{\prime} t^{\prime}\right)^{p r}=A^{p^{r}} t^{p^{r}} t_{1}^{p^{r(p r-1) / 2}}
$$

since $t_{1}^{\prime}$ is commutative with both $A^{\prime}$ and $t^{\prime}$. Hence

$$
s^{-1} A^{p^{r}} s=A^{p^{r}} t^{p^{r}} t_{1}^{p^{r}\left(p^{r-1) / 2}\right.} t_{2},
$$

where $t_{2}$ is contained in $H_{j-2}$. But $A^{p^{r}}$ is contained in $H_{k-1}$ and is therefore commutative with $s$. Hence

$$
t^{p^{r}} t_{1}^{p^{r}\left(p^{r}-1\right) / 2} t_{2}=1
$$

This implies that $t^{p^{r}}=1$ since $t_{1}^{p r\left(p^{r-1) / 2}\right.} t_{2}$ is contained in $H_{j-2}$ and $t^{p^{r}}$ cannot be contained in $H_{j-2}$ unless this is composed of the identity alone. Since then the order of the commutator $t$ does not exceed $p^{r}, t$ must be invariant in $G$. Hence no operation of $H_{k-1}$ can give a non-invariant commutator and $H_{k-1}$ must be contained in $H_{2}$. This requires that $k \leqq 3$.

Suppose now that $H_{k-1}$ is of class $k_{1}(>1)$ and assume that $k \leqq 2 j+1$ when $H_{k-1}$ is of class $j$ and $j<k_{1}$. We have just seen that this assumption is valid when $H_{k-1}$ is abelian. Every $\left(k_{1}-1\right)$ th commutator of $H_{k-1}$ is invariant in $H_{k-1}$. If we take any such commutator for the $s$ of the argument just used for the case $j=1$, we shall see that this commutator must be contained in $\mathrm{H}_{2}$. Hence the class of the corresponding central of $G / H_{2}$ is 
less than, or equal to, ${ }^{*} k_{1}-1$. But $G / H_{2}$ is of class $k-2$, and therefore in accordance with our assumption $k-2 \leqq 2\left(k_{1}-1\right)+1$, or $k \leqq 2 k_{1}+1$.

In general, if $H_{k-l}(l>1)$ is abelian, let $s$ be any one of its operations and $A$ any operation of $G$ not contained in $H_{k-l}$. Then

$$
A^{-1} s A=s t,
$$

where $t$ is contained in, say, $H_{j}$, but not in $H_{j-1}$. If

$$
A^{-1} t A=t t_{1}
$$

and

$$
A^{-1} t_{n} A=t_{n} t_{n+1} \quad(n=1,2, \cdots),
$$

then

$$
A^{-p^{l r}} s A^{p^{l r}}=s t^{p^{l r}} t_{1}^{p l r\left(p^{l r}-1\right) / 2} \cdots t_{n}^{x} \cdots t_{n_{1}}^{y},
$$

when $x$ equals the coefficient of the $(n+2)$ th term in the expansion of the $p^{l r}$ th power of a binomial and $y=1$ or the coefficient of the $\left(n_{1}+2\right)$ th term in this expansion, according as $n_{1}=p^{l r}-1$ or as $t_{n_{1}}$ is the first of this series of commutators with which $A$ is commutative. Now $H_{j-n}$ contains $t_{n}$ and $H_{j-l-1}$ contains

$$
t_{1}^{p l r(p l r-1): 2} \cdots t_{n}^{x} \cdots t_{n_{1}}^{y}
$$

but not $t^{p^{l r}}$, unless $H_{j-l}$ is composed of the identity alone. But $A^{p^{l r}}$ is commutative with $s$. Hence $t^{p^{l r}}=1$. It follows from this that $H_{k-l}$ must be contained in $H_{l+1}$, and $k-l \leqq l+1$, or $k \leqq 2 l+1$. Suppose now that $H_{k-l}$ is of class $k_{l}(>1)$ and assume that $k \leqq j l+j+l$ when $H_{k-l}$ is of class $j$ and $j<k_{l}$. We have just seen that this assumption is valid when $H_{k-l}$ is abelian. Every $\left(k_{l}-1\right)$ th commutator of $H_{k-l}$ is invariant in $H_{k-l}$, and can therefore give in $G$ only commutators whose orders do not exceed $p^{l r}$. Every such $\left(k_{l}-1\right)$ th commutator must therefore be contained in $H_{l+1}$. Hence the class of the central of $G / H_{l+1}$ that corresponds to $H_{k-l}$ is less than, or equal to, $k_{l}-1$. But $G / H_{l+1}$ is of class $k-l-1$, and therefore in accordance with our assumption $k-l-1 \leqq\left(k_{l}-1\right) l+k_{l}-1+l$, or $k \leqq k_{l} l+k_{l}+l$. Hence

If $G$ is of order $p^{m}$ ( $p$ an odd prime) class $k$ and if every commutator of $G$ that is contained in $\mathrm{H}_{2}$ and whose order does not exceed the highest order of any invariant commutator is itself an invariant commutator, then $H_{k-l}$ is of class $k_{l}$, where $k \leqq k_{l} l+k_{l}+l$.

Groups with cyclic commutator subgroups and of order $p^{m}$ are included in the category considered here.

Columbia University,

November, 1914.

* Fite, loc. cit. 